\title{
NEAR THRESHOLD FATIGUE CRACK GROWTH SIMULATION
}

\author{
T. Meshii ${ }^{1}$, K. Ishihara ${ }^{2}$ and K. Watanabe ${ }^{3}$ \\ ${ }^{1}$ Professor, Graduate School of Engineering, University of Fukui, Bunkyo, Fukui, Fukui, 910-8507, Japan \\ ${ }^{2}$ Graduate Student, Graduate School of Engineering, University of Fukui, Bunkyo, Fukui, Fukui, 910-8507, Japan \\ ${ }^{3}$ Professor, Institute of Industrial Science, University of Tokyo, Komaba, Meguro-ku, Tokyo, 153-8505, Japan
}

\begin{abstract}
In this paper, we performed the numerical simulations by the proposed simplified model to explain the decrease in threshold stress intensity factor (SIF) range $\Delta K_{\text {th }}$ due to high maximum SIF $K_{\max }$. The results with tentative material resistances showed the validity of the proposed model, at least in the meaning that the decrease in $\Delta K_{\text {th }}$ by the increase with $K_{\max }$ was simulated.
\end{abstract}

\section{INTRODUCTION}

Since the pioneering work of Paris and Erdogan [1], the applied stress intensity factor (SIF) range $\Delta K$ has been known to be a major controlling parameter in fatigue crack growth (FCG) rate $d a / d N$ under small scale yielding conditions. At low FCG rates, $d a / d N-\Delta K$ curve in log-log scale generally becomes steep and appears to approach a vertical asymptote that corresponds to the FCG threshold. $\Delta K$ corresponding to this asymptote is named as the threshold SIF range $\Delta K_{\text {th. }}$ Almost without exception, existing data show that the $\Delta K_{\text {th }}$ tends to decrease with increasing load ratio $R$ [2]. Schmidt and Paris rationalized this behavior solely on the basis of the crack closure concept [3]. According to their model, we expect that the $\Delta K_{\text {th }}$ obtained by the $K_{\max }$-constant test method (that ensures closure free conditions) is independent of $K_{\max }$, and that it is constant. However, the decrease in $\Delta K_{\text {th }}$ due to high $K_{\max }$ is reported for some materials tested with $K_{\max }$-constant methods [2]. To explain this decrease in $\Delta K_{\text {th }}$ due to high $K_{\max }$, in this paper, we numerically modeled our qualitative model and ran numerical simulations to show the validity of our model.

\section{SIMULATIONS}

\subsection{FCG algorism}

From the observation of SEM fractographs and the assumptions we have made, we consider that the FCG mechanism is the same regardless of $\Delta K$, based on the standpoint that microscopic cracks grow locally (such as in crystal grain) due to a cyclic or static mode fracture and the macroscopic crack front growth is observed as coalescences of microscopic cracks. In this case, the microscopic crack growth in any direction will contribute to macroscopic crack growth, because the crystal grain is separated [4]. Fig. 1 is a simplified model showing this mechanism, applied to FCG in an ASTM CT specimen. 
In Fig. 1, each square (hereafter called as a cell) corresponds to a grain. We specify the location of a cell, for example, as $i$ th row and $j$ th column ( $\left.i=1 \sim n_{\text {row }}, j=1 \sim n_{\text {column }}\right)$. In our model, we assume that the microscopic crack growth mechanism is selectively determined as cyclic or static mode failure, depending on the direction of the slip plane of the grain. Here we specify material resistances for cyclic and static

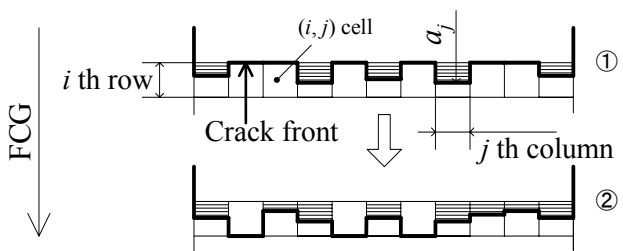

Figure 1: Our simplified mesoscopic crack coalescence FCG model [4] mode failure as $\Delta k_{\mathrm{S} i j}$ and $k_{\mathrm{C} i j}$, respectively. Another simplification is that the microscopic crack growth direction coincides with the macroscopic FCG direction. The depth of $j$ th column is $a_{j}$ and crack front is generally uneven as shown in Fig.1. The nominal load in the simulation is controlled so that the maximum SIF is equal to the specified $K_{\max }$ and so that the SIF range $\Delta K$ - average crack length $a=\left(a_{1}+a_{n \text { column }}\right) / 2$ relationship for CT specimen

$$
\Delta K=\Delta K_{0} \exp \left(C\left(a-a_{0}\right)\right)
$$

holds, same as that for standard ASTM FCG tests [5]. Here $\Delta K_{0}$ is $\Delta K$ corresponding to the initial crack length $a_{0}$ and $C$ is the $\Delta K$-gradient, respectively.

Because of the uneven crack front with local length $a_{j}$, the local crack driving force in the $j$ th column is distributed so that the maximum SIF and SIF range becomes $k_{j}, \Delta k_{j}$, respectively. Then these crack driving forces in the $j$ th column are compared with the material resistances of the crack tip cells. Criteria we apply in our simulation is that there is a local crack growth in the case of $\Delta k_{j}>$ $\Delta k_{\mathrm{S} i j}$ or $k_{j}>k_{\mathrm{C} i j}$, and on the other hand no local crack growth if $\Delta k_{j}<\Delta k_{\mathrm{S} i j}$ and $k_{j}<k_{\mathrm{C} i j}$. When $\Delta k_{j}<$ $\Delta k_{\mathrm{S} i j}$ and $k_{j}<k_{\mathrm{C} i j}$ is satisfied for all columns, we finish the simulation and the nominal SIF range $\Delta K$ at this time is named as $\Delta K_{\text {th }}$. The concrete algorism of the FCG simulation is shown as a flow chart in Fig. 2. In the following, detailed procedures of the simulation are explained step by step, referring to Fig. 2.

\subsubsection{Initial Setting}

First, we specify CT specimen dimensions ( $B$ : thickness, $W$ : width, $a_{0}$ : initial crack length, $a_{\mathrm{END}}$ : crack length for forced termination), quantities for specifying the size of a cell and its location ( $g$ : size of one cell in FCG direction, $n_{\text {row }}, n_{\text {column }}$ ), quantities relevant to material properties ( $E$ : Young's modulus, v. Poisson's ratio, $C_{\text {Paris, }} m$ :

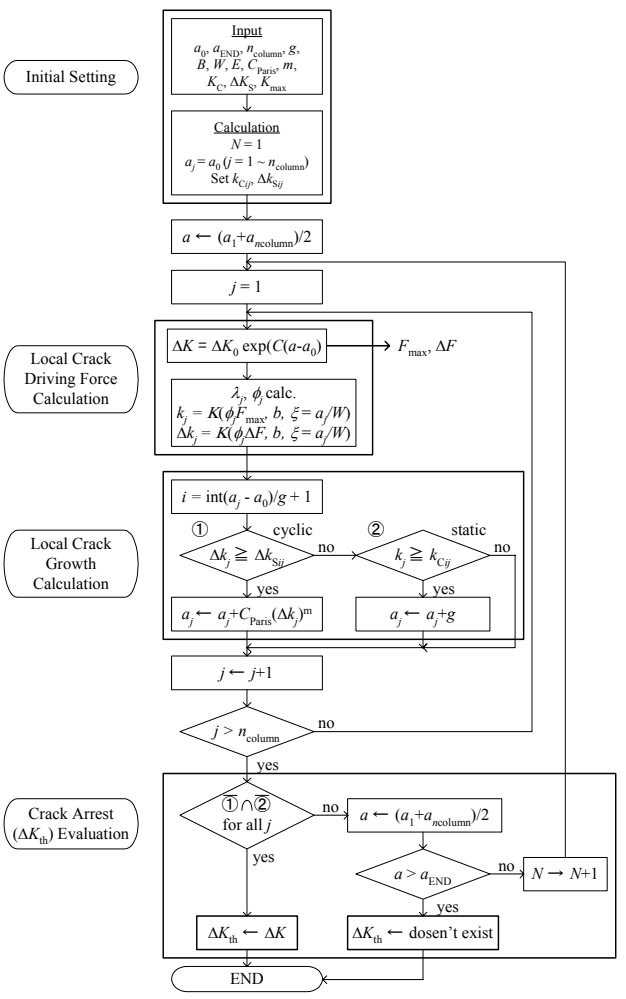

Figure 2: Flow chart of FCG algorithm material constants of Paris law, $K_{\mathrm{C}}, \Delta K_{\mathrm{S}}$ : basic material resistances for static and cyclic mode failure, respectively, $\alpha, \beta$ : the parameters to specify the scatter in material resistance of each cell, $R I$ : uniform random number seed).

Then the material resistances of each cell are randomly distributed by the following formula 
using the uniform random numbers $\psi_{i j 1}, \psi_{i j 2}$ of $0 \sim 1$ generated with $R I$.

$$
\begin{aligned}
k_{C i j} & =\left(\alpha+\beta \psi_{i j 1}\right) K_{\mathrm{C}} \equiv \Psi_{i j 1} K_{\mathrm{C}} \\
\Delta k_{\mathrm{S} i j} & =\left(\alpha+\beta \psi_{i j 2}\right) \Delta K_{\mathrm{S}} \equiv \Psi_{i j 2} \Delta K_{\mathrm{S}}
\end{aligned}
$$

\subsubsection{Local Crack Driving Force Calculation}

As we described in section 2.1, the nominal load in the simulation is controlled so that the maximum SIF is equal to the specified $K_{\max }$ and so that the SIF range $\Delta K$ - average crack length $a$ $=\left(a_{1}+a_{n \text { column }}\right) / 2$ relationship for CT specimen satisfies eqn (1). The nominal maximum load $F_{\max }$ and the load range $\Delta F$ corresponding to the nominal these crack driving forces can be counted backward from the SIF formula for the ASTM's standard CT specimen [5].

$$
K(F, B, \xi=a / W)=\frac{F}{B \cdot W^{0.5} \cdot(1-\xi)^{1.5}}\left[(2+\xi) \cdot\left(0.886+4.64 \cdot \xi-13.32 \cdot \xi^{2}+1472 \cdot \xi^{3}-5.6 \cdot \xi^{4}\right)\right]
$$

Then, in order to calculate the local crack driving force $k_{j}$ and $\Delta k_{j}$ in the crack tip of $j$ th column, the load distribution coefficient $\phi_{j}$ is calculated by modeling the $\mathrm{CT}$ specimen with $n_{\text {column }}$ slices of width $b=B / n_{\text {column, }}$ crack length $a_{j}$ and compliance $\lambda_{j}$ as shown in Fig. 3. Since the slice model can be considered as a parallel spring,

$$
\phi_{j}=1 /\left(\lambda_{j} \sum_{l=1}^{n_{\text {olumu }}}\left(1 / \lambda_{l}\right)\right)
$$
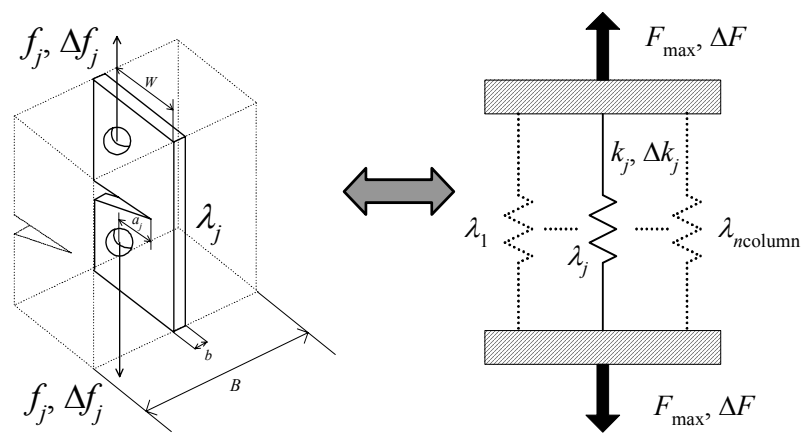

Figure 3: Slice model for stress redistribution

Here we used ASTM's following equation [5] for evaluation of $\lambda_{j}\left(\xi=a_{j} / W\right)$,

$$
\lambda_{j}=\frac{1-v^{2}}{E b}\left\{\frac{19.75}{(1-\xi)^{2}}\left(0.5+0.192 \xi+1.385 \xi^{2}-2.919 \xi^{3}+1.842 \xi^{4}\right)\right\}
$$

Since the maximum load and the load range of the local load of the slice of $j$ th column is $\phi_{j} F_{\max }$ and $\phi_{j} \Delta F$, respectively, $k_{j}$ and $\Delta k_{j}$ can be calculated by the eqn (4) as follows.

$$
\begin{aligned}
& k_{j}=K\left(\phi_{j} F_{\max }, b, \xi=a_{j} / W\right) \\
& \Delta k_{j}=K\left(\phi_{j} \Delta F, b, \xi=a_{j} / W\right)
\end{aligned}
$$

\subsubsection{FCG Calculation}

Once the local crack driving force of $j$ th column is obtained, crack growth is evaluated for each column. First, row index $i$ of the crack tip cell is evaluated using the material resistances for this cell. Then occurrence of local crack growth is judged by comparing the local crack driving force $\Delta k_{j}, k_{j}$ with the material resistances of this cell $\Delta k_{\mathrm{S} i j}, k_{\mathrm{C} i j}$ for all columns. Naturally, in this evaluation, we cannot evaluate the local crack growths by cyclic and static modes at the same time. If we simulate the load cycle faithfully, an idea to evaluate the crack growth by static mode and then that by cyclic mode might be considered better. However, it is reported that damage due to static mode failure does not necessarily occur in case slip occurs early in a load cycle [6]. Thus, we chose to first evaluate the occurrence of the cyclic mode failure and, in case of no cyclic mode failure, then evaluate the occurrence of static mode failure. We evaluated the amount of local crack growth in a certain cycle as follows: 
(1) In case of $\Delta k_{j}>\Delta k_{\mathrm{S} i j}$, the amount of local crack growth in the $j$ th column is evaluated as $C_{\text {Paris }}\left(\Delta k_{j}\right)^{m}$ according to the Paris law.

(2) In case of $k_{j}>k_{C i j}$, the amount of local crack growth is assumed to be $g$, the size of a cell. (Note: There is a possibility that crack growth due to static failure mode is smaller than $g$, as a result that $k_{j}>k_{\text {Cij }}$ becomes not satisfied due to load redistribution. However, change in load distribution factor $\phi_{j}$ due to local crack growth calculated by eqn (4) was small, so we simply evaluated the amount of growth as $g$ ).

\subsubsection{Crack Arrest Evaluation}

Finally, crack arrest evaluation is performed after local crack growth evaluation is performed for all columns.

(1) In case $\Delta k_{j}<\Delta k_{\mathrm{S} i j}$ and $k_{j}<k_{\mathrm{C} i j}$ are satisfied for all columns, we define that the crack has arrested. The nominal SIF range $\Delta K$ at this time is the $\Delta K_{\text {th }}$ that we wanted to obtain.

(2) In case $a>a_{\text {END }}$ is satisfied as a result of FCG, we define that there was no $\Delta K_{\text {th }}$.

If these conditions are not satisfied, local crack driving force calculation is again performed for the next cycle.

\subsection{Preliminary calculation conditions and results}

We carried out the simulation corresponding to our $K_{\max }$-constant test for JIS carbon steel S55C by CT specimen whose size is $B=12.5, W=50$, and $a_{0}=18 \mathrm{~mm}$.

The size of a cell was set to $g=0.03 \mathrm{~mm}$ because the grain size was approximately that size. From this $g$ and $B$, we set $n_{\text {column }}=400$. Considering that the total amount of crack growth in a test was about $5 \mathrm{~mm}$, we set $n_{\text {row }}=168$. Crack length for simulation termination was set as $a_{\mathrm{END}}=21$ $\mathrm{mm}$. As material constants, $E=206 \mathrm{GPa}, v=0.3$, and $C_{\text {Paris }}=1.35 \times 10^{-9}, m=3.77$ obtained from tests [4] were used. The average value SIF for which the CT specimens experienced forced fracture was used as the basic material resistance $K_{\mathrm{C}}$. On the other hand, $\Delta K_{\mathrm{S}}$ was set as $3 \mathrm{MPam}^{1 / 2}$ because test results of $\Delta K_{\text {th }}$ for low $K_{\max }$ was that value. Parameters to specify the scatter in material resistance of a cell was tentatively set as $\alpha=0.5$ and $\beta=1.0$ (namely, $\psi_{i j 1}$ and $\psi_{i j 2}=0.5 \sim$ 1.5).

Constants in eqn (1) for load control was set as $\Delta K_{0}=12 \mathrm{MPam}^{1 / 2}$ and $C=-0.7 \mathrm{~mm}^{-1}$, that were used for our tests [4].

Here we tentatively specified the basic material resistances as aforementioned, though there are various possible combinations. Thus we first considered the influence of $K_{\mathrm{C}} / \Delta K_{\mathrm{S}}$ on $d a / d N$ and $\Delta K_{\mathrm{th}}$, under condition of identical scatter in material resistances $\left(\psi_{i j 1}\right.$ and $\psi_{i j 2}$ for $\left.R I=81\right)$. The results showed that $\mathrm{K}_{\mathrm{C}} / \Delta \mathrm{K}_{\mathrm{S}}$ had no effect on $d a / d N$ - $\Delta K$ curve nor $\Delta K_{\mathrm{th}}$, when $K_{\max } / K_{\mathrm{C}}$ was set to a same value. Thus, the effect of $K_{\max }$ on $d a / d N-\Delta K$ curve and $\Delta K_{\text {th }}$ was considered with constant $K_{\mathrm{C}} / \Delta K_{\mathrm{S}}$ hereafter.

The results are shown in Fig. 4 and 5. In Fig. 4, FCG rate $d a / d N$ was evaluated by the incremental polynomial method given in ASTM

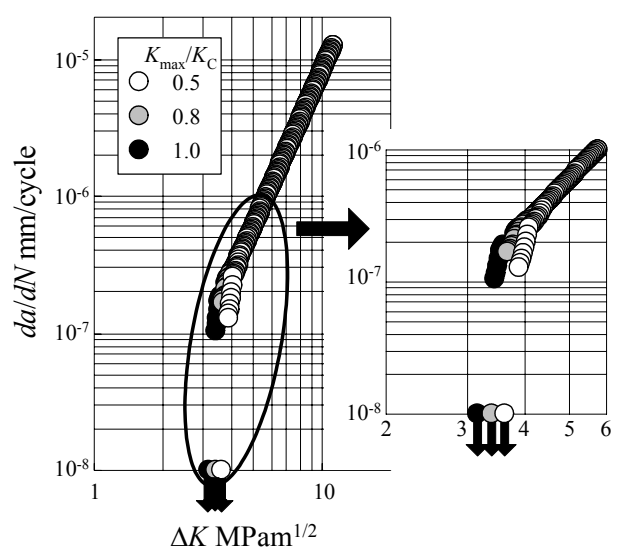

Figure 4: $K_{\max }$-const. simulation results 
E647 [5]. The $\Delta K$ corresponding to a plot for $d a / d N=10^{-8} \mathrm{~mm} /$ cycle is the nominal $\Delta K$ when the conditions of $\Delta k_{j}<\Delta k_{\mathrm{S} i j}$ and $k_{j}<k_{\mathrm{C} i j}$, were satisfied. This $\Delta K$ is called $\Delta K_{\text {th }}$ in this simulation.

We see from Fig. 4 that the $d a / d N-\Delta K$ curves coincides for three $K_{\max } / K_{\mathrm{C}} \mathrm{S}$ in the range of $10^{-6} \sim 10^{-5}$ $\mathrm{mm} /$ cycle. On the other hand, we see the decrease in $\Delta K_{\text {th }}$ due to high $K_{\max }$ in the range below $d a / d N=10^{-6} \mathrm{~mm} /$ cycle. Thus we conclude that we can simulate the decrease in $\Delta K_{\text {th }}$ due to high $K_{\max }$ by our model [4], that was originally proposed to qualitatively explain the phenomenon observed for some materials under
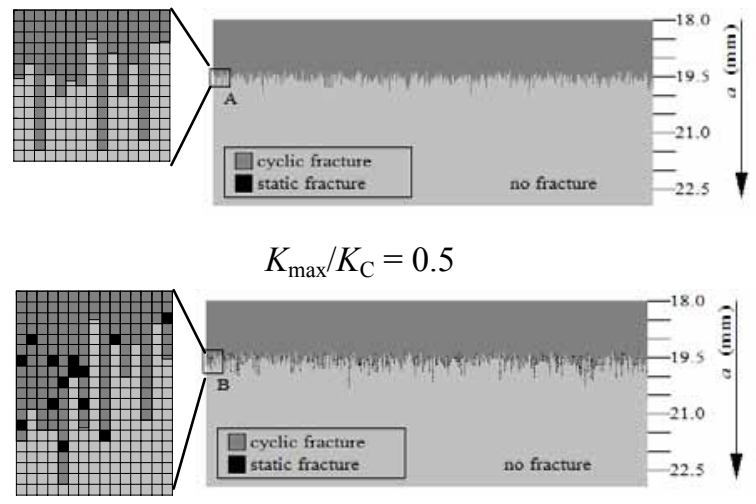

$$
K_{\max } / K_{\mathrm{C}}=1.0
$$

Figure 5: Fracture surface for Fig. 4 $K_{\text {max }}$-constant $\Delta K_{\text {th }}$ tests.

Fig. 5 is the fractured surfaces at the simulation end, corresponding to Fig. 4. The light gray, intermediate gray and black colored cell represent the no-fractured, cyclic-mode and static mode fractured cell, respectively. Digits with the scale on the right hand of the figure shows the crack length. We see in the cases of $K_{\max } / K_{\mathrm{C}}=1.0$ that static mode fractured cells have appeared for $a>$ $19.5 \mathrm{~mm}$. On the other hand, in the case of $K_{\max } / K_{\mathrm{C}}=0.5$, we see from Fig. 5 that the crack growth by the static mode failure has not occurred.

\subsection{Detailed examination of the decrease in $\Delta K_{\text {th }}$ due to high $K_{\max }$}

Since phenomenon of the decrease in $\Delta K_{\text {th }}$ was observed when $K_{\max }$ was increased as $K_{\max } / K_{\mathrm{C}}=$ $0.5,0.8$ and 1.0 in the aforementioned, we increased the numbers of simulations to examine this phenomenon in detail. We made simulations for six $K_{\max } / K_{\mathrm{C}}$ in the range of $0.5 \sim 1.0$, graduated in 0.1 increments. For each $K_{\max } / K_{\mathrm{C}}, 1352$ material resistance distribution was considered by varying the uniform random number seed $R I$. For all cases, scatter in material resistance of a cell was tentatively set as $\alpha=0.5$ and $\beta=1.0\left(\psi_{i j 1}, \psi_{i j 2}=0.5 \sim 1.5\right)$, identical with the aforementioned. The results are summarized as Fig. 6 .

In Fig. 6, a closed circle with bars on upper and lower side represents the average $\mu \pm$ standard deviation $\sigma$ of $\Delta K_{\mathrm{th}} / \Delta K_{\mathrm{S}}$ obtained from 1352 material resistance distributions. Maximum and the minimum values of $\Delta K_{\mathrm{th}} / \Delta K_{\mathrm{S}}$ are also shown in the figure for reference. We first see from the figure that the decrease in $\Delta K_{\text {th }}$ due to high $K_{\max }$ appears generally from the simulation and that the results in Fig. 4 were not special ones. Another finding is that the average value of $\Delta K_{\mathrm{th}} / \Delta K_{\mathrm{S}}$ seems to decrease linearly due to the increase in $K_{\max } / K_{\mathrm{C}}$. This linear relationship has been reported for carbon

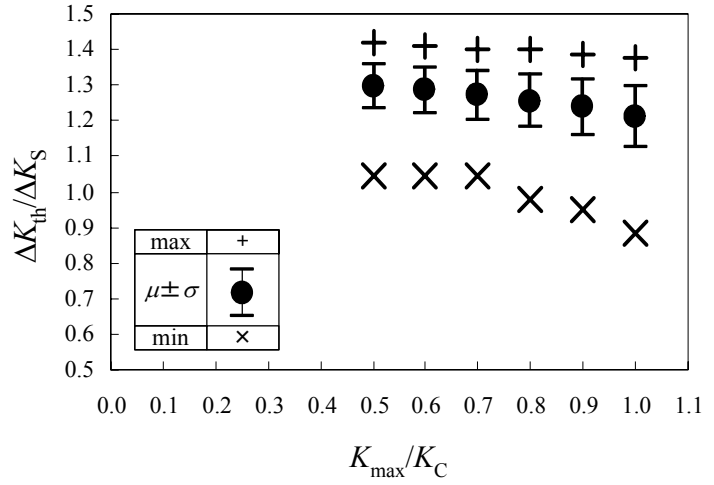

Figure 6: Relation of $K_{\max } / K_{\mathrm{C}}$ and $\Delta K_{\mathrm{th}} / \Delta K_{\mathrm{S}}$ $\left(\alpha=0.5, \beta=1.0, \Psi_{i j 1}, \Psi_{i j 2}=0.5 \sim 1.5\right)$ 
steel S55C [4] and for Ti and Al alloys [2].

In this meaning, though we used tentative values and made many assumptions, we conclude that our simplified FCG model [4] can simulate the decrease in $\Delta K_{\text {th }}$ due to high $K_{\max }$.

\section{DISCUSSION}

As aforementioned in section 2.1, the local crack tip damage evaluation procedure was to first evaluate the occurrence of the cyclic mode failure, and in case of no cyclic mode failure, then evaluate the occurrence of static mode failure. As a result, the damage mechanism of a fractured cell is limited to the cyclic mode failure in the range of $\Delta K / \Delta K_{\mathrm{S}}>1.4$ (about 1.5, $a<19.5 \mathrm{~mm}$ ), because a cell satisfying $\Delta k_{j}<\Delta k_{\mathrm{Sij}}$ does not appear when scatter in the material resistance is set as $\psi_{i j 1}, \psi_{i j 2}=0.5 \sim 1.5$. On the contrary, the evidence of a static mode failure has been found in the corresponding region of the actual specimen, although the number was few [4]. In the present simulation, we paid attention to FCG near the $\Delta K_{\text {th }}$, and assumed that the static mode failure was an additional damage as a first trial. We admit that improvement is still necessary on selective damage algorithm.

In Fig. 6, we see that the average value of $\Delta K_{\mathrm{th}} / \Delta K_{\mathrm{S}}$ at a specific $\mathrm{K}_{\max } / \mathrm{K}_{\mathrm{C}}$ was in the range of $1.2 \sim 1.3$ when $K_{\max } / K_{\mathrm{C}}=0.5 \sim 1.0$. Though not shown in the figure, $\Delta K_{\mathrm{th}} / \Delta K_{\mathrm{S}}$ was approximately 1.3 for $K_{\max } / K_{\mathrm{C}}<0.5$. In addition, the minimum $\Delta K_{\mathrm{th}} / \Delta K_{\mathrm{S}}$ for $K_{\max } / K_{\mathrm{C}} \leq 0.5$ was larger than unity. One might have expected the single value of unity instead for this $K_{\max } / K_{\mathrm{C}}$. At this moment, we do not have a definite answer to explain the discrepancy between the simulation result and the expected value. However, we know from some additional simulations with smaller scatter in the material resistance that this average $\Delta K_{\mathrm{th}} / \Delta K_{\mathrm{S}}$ decreases from 1.3. We are still continuing investigation for this subject.

In our simulation, scatter in material resistances were tentatively set as $\Psi_{i j 1}, \Psi_{i j 2}=0.5 \sim 1.5$. Our test results for the $\mathrm{S} 55 \mathrm{C}$ show that $\psi_{i j 1}$ (variation of $K_{\mathrm{C}}$ ) is $0.95 \sim 1.03$ to average value, and $\psi_{i j 2}$ (variation in $\Delta K_{\text {th }}$ to a specific $K_{\max }$ ) is $0.93 \sim 1.07$ to average value [4]. In this meaning, the scatter in material resistances we used might be considered to be overestimating. However, to apply $K_{\mathrm{C}}$ or $\Delta K_{\text {th }}$ as material constants itself is an assumption. Thus, basic material resistances coupled with an appropriate scatter has to be discussed further to refine the model.

\section{CONCLUSION}

In this paper, we performed the numerical simulations by the proposed simplified model to explain the decrease in threshold stress intensity factor (SIF) range $\Delta K_{\text {th }}$ due to high maximum SIF $K_{\max }$. The results with tentative material resistances showed the validity of the proposed model, at least in the meaning that the decrease in $\Delta K_{\text {th }}$ by the increase with $K_{\max }$ was simulated.

\section{REFERENCES}

[1] Paris, P.C. and Erdogan, F., Trans. ASME, Ser. D, Vol. 85, pp. 528-534, 1963.

[2] Boyce, B.L. and Ritchie, R.O., Engineering Fract. Mechanics, Vol. 68, pp. 129-147, 2001.

[3] Schmidt, R.A. and Paris, P.C., ASTM STP 536, pp. 79-94, 1973.

[4] Meshii, T., Ishihara, K. and Watanabe, K., Assessment for Decrease in Threshold Stress Intensity Factor (SIF) Range due to High Maximum SIF, Trans. JSME (A), in Japanese, Vol. 70, pp. 604-611, 2004.

[5] ASTM, Standard test Method for Measurement of Fatigue Crack Growth Rate, Specification E647-93, American Society for Testing and Materials, Philadelphia, pp. 577-613, 1999.

[6] Ritchie, R.O. and Knott, J.F., Acta Metallurgica, Vol. 21, pp. 639-648, 1973. 